\title{
Pilot study of testing a clinical tool for pelvic physical examination in patients with vulvodynia
}

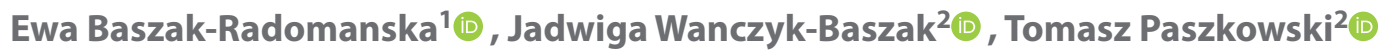 \\ ${ }^{1}$ Terpa, Lublin, Poland \\ ${ }^{2} 3^{\text {rd }}$ Chair and Department of Gynecology, Medical University in Lublin, Poland
}

\begin{abstract}
Objectives: Vulvodynia diagnosis is based on medical history and physical examination. The study is aimed to evaluate the clinical usefulness of a pelvic floor physical examination (VAMP protocol) for vulvodynia diagnosis, applied during gynecological examination, proposed as educational and diagnostic tool.

Material and methods: Pelvic physical examinations were performed for 650 non-pregnant female patients. A study group of 449 cases met the vulvodynia diagnostic criteria (120 with provoked, 104 with spontaneous, and 121 with mixed subtype) and were compared with those of 201 healthy individuals. Four anatomical regions were examined: the vulva $(\mathrm{V})$ and anus (A) with a cotton swab, the internal pelvic muscles (M) with a digital examination of the levator ani, and the paraurethral $(\mathrm{P})$ area with digital pressure. Only the maximum pain score for a given area was recorded, using a Numerical Rating Scale. The four anatomical regions were recorded under the VAMP acronym.

Results: Differences in mean scores VAMP protocol were statistically between vulvodynia and comparison group for $V=6.48$ vs $0.98 ; M=6.29$ vs 1.05 ; and $P=6.89$ vs 1.33 , with exception of $A=0.03$ vs 0.08 . Patient age, weight, way of delivery, other concomitant diseases (e.g., dysuria, anal and bowel symptoms), vulvodynia subtype, and pain duration did not influence VAMP scores in patients with vulvodynia and comparison group.

Conclusions: Pelvic examination according to VAPM protocol can be applied in vulvar pain patients for diagnostic purposes. Besides of vulvodynia symptoms any other analyzed variables did not influence on scores of VAMP protocols. We found that cut-off score $\geq 3$ even in one of $\mathrm{V}, \mathrm{M}$ or $\mathrm{P}$ component of VAMP protocol can be considered as diagnostic criterium for vulvodynia. Component $\mathrm{A}$ (anus area) was not useful for vulvodynia diagnosis.
\end{abstract}

Key words: gynecological exam; pelvic examination; pelvic floor muscles; vulvar pain; vulvodynia

Ginekologia Polska 2021; 92, 6: 410-416

\section{INTRODUCTION}

Vulvodynia (Vd) is a form of chronic vulvar pain and other discomfort that persists for more than three months in the absence of any evident vulvovaginal pathology, in accordance with the criteria outlined in the recent consensus statement [1]. Chronic vulvar pain is most commonly described as pain perceived superficially in areas of the vulva, anus, perineum, or vagina [2-4]. The International Society for the Study of Vulvovaginal Disease (ISSVD) described Vd as vulvar discomfort, reported as pain, irritation, pruritus, sickness, and oversensitivity, which are often overlooked [1, 2]. Remission and relapse are common, although consecutive recurrences can present varying symptoms that can be confusing for the patient and healthcare practitioners.
The classification of vulvodynia is based on the site of the pain; whether it is generalized, localized, or mixed; whether it is provoked (pain on touching, pressure, vaginal penetration) or spontaneous (for no apparent reason), or mixed; whether the onset is primary or secondary; and the temporal pattern (whether the pain is intermittent, persistent, constant, immediate, or delayed) [1, 2] .

In the general population, $\mathrm{Vd}$ affects $8.3-16 \%$ of adult women $[2,5,6]$, and the most prevalent form is provoked $\mathrm{Vd}$, which presents in $12 \%$ of women and can cause superficial dyspareunia. Spontaneous Vd is less common (6-7\% of $V d)$ [5].

$\mathrm{Vd}$ is classified as a functional pain syndrome that is mediated by pelvic floor muscles (PFM) dysfunction in the

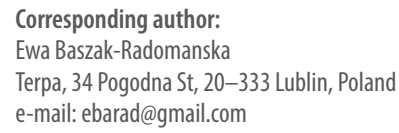

This article is available in open access under Creative Common Attribution-Non-Commercial-No Derivatives 4.0 International (CC BY-NC-ND 4.0) license, allowing to download articles and share them with others as long as they credit the authors and the publisher, but without permission to change them in any way or use them commercially. 
form of an overactive, hypertonic/non-relaxing muscle state that then potentially gives rise to pain [3,5-11]. Overactive muscles have been suspected in $80-90 \%$ of patients with $\mathrm{Vd}$ and provide the indication for pelvic manual and general physiotherapy $[12,13]$. A psychologic predisposition to chronic functional pain syndromes has been previously identified [14], and emotional stress can contribute to myofascial disorders. The manual palpation of muscles and points of tenderness (trigger points) provides an effective real-time method for identifying the source of pain in $\mathrm{Vd}$ $[9,15-17]$, although there is no standardized protocol for clinical practice [18]. From the literature, several key points should be maintained for pelvic physical exam requirements in patients with chronic pain [18]. The Numeric Rating Scale (NRS) is a preferred scale for patient self-perceived pain ratings $[9,19-21]$, graded during the test using a numeric scale of 0 to 10 , with 0 representing "no pain at all" and 10 suggesting "the worst possible pain".

Diagnosis of $\mathrm{Vd}$ is based on medical history (reported symptoms, duration of pain or other discomfort), vulvar examination with exclusion of other specific disorders as a cause of pain, like identified infection (e.g., candidiasis, herpes), inflammation (e.g., lichen simplex chronicus, sclerosus, lichen planus), neoplasia (eg, Paget disease, vulvar cancer), or a neurologic disorder (e.g., herpes neuralgia) [1]. Consecutive step in Vd diagnosis is composed of hymenal remnant base pain on pressure as a cotton swab testing $[9,15]$. Vestibular cotton swab test remains PFM referred pain anatomical area, what is sufficient for Vd diagnosis [22]. However, examinations of additional anatomical areas can give additional, accurate insight to increase internal consistency in PFM overactive status assessment in Vd women [15-17]. Examination of pelvic muscles is recommended but rarely conducted. Currently no standardized protocol exists $[9,15,17,18]$.

The Integrated Pain Mapping and Assessment Protocol (IMAP) was developed for research purposes as referred pain diagnostic maps by Jantos [13, 19], and includes 54 relevant and irrelevant, external and internal points of examination. Shortened version of IMAP, which focuses on the vulva, anus, internal pelvic muscles, and paraurethral regions was elaborated, proposed as the part of gynecological examination, recorded as VAMP acronym and evaluated in this study as a simplified clinical pain mapping modality for diagnostic and educational purposes. The study is aimed to evaluate the clinical usefulness of a pelvic floor physical examination (VAMP protocol) for vulvodynia diagnosis, applied during gynecological examination, proposed as educational and diagnostic tool.

\section{MATERIAL AND METHODS}

The study characteristics were extracted from the medical records at outpatient clinic for women, with a focus on vulvar diseases. The study was approved by the appro- priate IRB. We included patients with Vd who were seen between January 2016 and July 2018. The described diagnostic method has been applied as a routine practice at the center since 2014. The comparative data was obtained from a group of healthy women between February 2019 and June 2019. Every patient in both groups were diagnosed by the study authors. A total of 650 women were included in the study, 449 of who met the diagnostic criteria for $\mathrm{Vd}$, as a study group, and 201 women were assigned to the control group. The included participants were nonpregnant adults, with no serious general diseases and urogenital surgery performed previously.

Vd was diagnosed as idiopathic vulvar pain (raw or vague) or other discomfort as burning sensation, stabbing, pressure, and pulling pain, also as the pruritus or dryness, discomfort, and oversensitivity. The symptoms sustained at least for three months, induced by vaginal penetration (provoked), without specific trigger (spontaneous) or both (mixed Vd subtype) [1, 2]. A general medical and gynecological history were recorded for every case, and a physical exam was carried out. Four anatomical regions were examined: the vulva (V) and anus (A) with a cotton swab test, the internal pelvic muscles $(\mathrm{M})$ with a digital examination of the levator ani, and the paraurethral $(P)$ area with digital pressure (Fig. 1). In every patient their VAMP scores were determined as a part of a gynecological exam, for any visible or detectable pathology exclusion. In the litothomy position vaginal speculum insertion, for gynecological purposes, was permitted to take place before the internal exam (when accepted or possible).

For the VAMP protocol, the following steps were followed consecutively (as outlined in Fig. 1):

- The Numerical Pain Rating Scale (NRS) was explained, through which the patients were asked to rate each examined point, if painful.

- Verbal consent should be taken after an explanation.

- A cotton swab test was performed by applying gentle pressure to five points within the vestibular base of the hymenal remnant (following a vestibular clock from the two to ten position) using a dry swob. The pressure was adjusted to a level tolerated by the patient. Only the maximum NRS rating was noted (V).

- A similar cotton swab test was then used for two points around the anus, with similar pressure as that used for the vulva, and the NRS maximum rating was noted (A).

- The insertion of one lubricated, gloved index finger, for a bimanual transvaginal or rectal examination was performed.

- The palmar side of the index finger was rotated backward to palpate the pelvic floor muscles. The finger was moved laterally along the length of the rectum, using the single sites mid-muscle belly technique, from the 


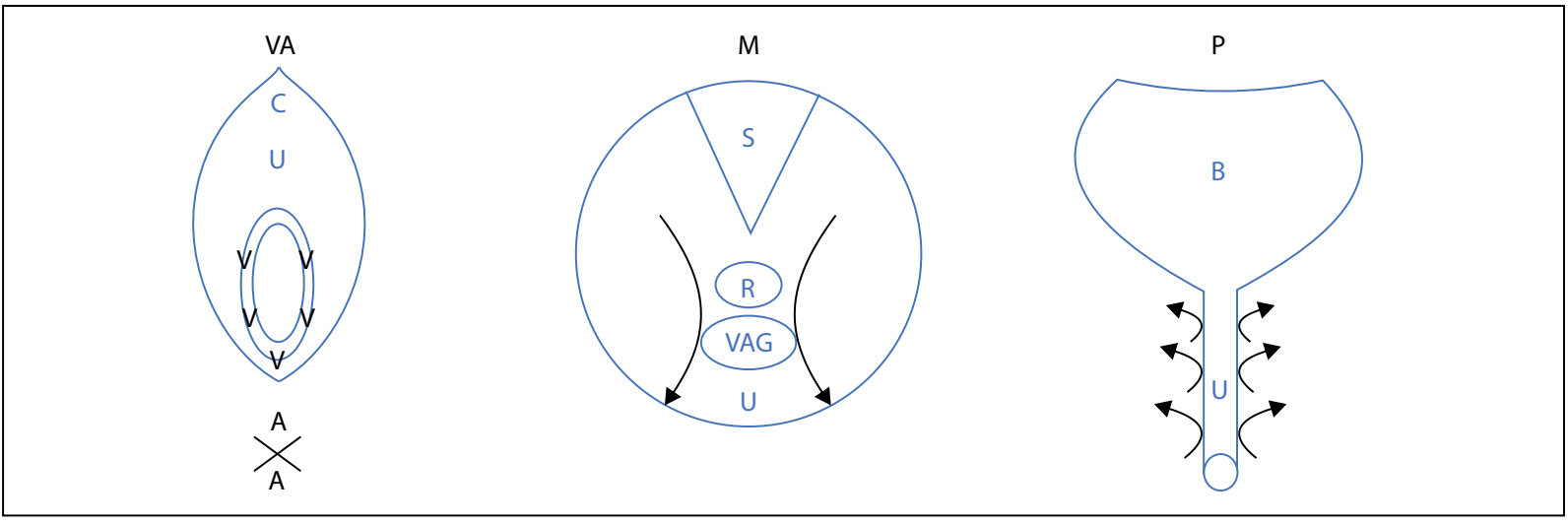

Figure 1. VAMP protocol diagram; $\mathrm{V}$ - vulva 5 points cotton swab pressure; $\mathrm{A}$ - anus 2 points cotton swab pressure; $\mathrm{C}-\mathrm{clitoris} ; \mathrm{U}-$ urethra; $\mathrm{S}$ - sacrum; $\mathrm{R}$ - rectum; VAG — vagina; B — bladder; arrows — internal digital examination

inside to the outside on both the right and the left side. A bilateral sliding motion was performed, with marked pressure applied to the muscles until the patient's accepted pain threshold was reached. The maximum NRS rating was noted $(\mathrm{M})$ in the medical records.

- For paraurethral area examination, the palmar side of the index finger was rotated upward, laterally to the urethra, and similarly to the point of urethra detachment from the pubic bone. The movement was performed from the outside to the inside of the pubis, lengthwise, on both the right and left sides. The pressure was increased with particular attention to patient pain tolerance, and the maximum NRS rating was noted $(\mathrm{P})$.

The physical exam results were recorded under the VAMP acronym (e.g., VAMP 3048), which reflects the maximum NRS ratings in the four areas: vulva, anus, muscles, and paraurethra. The pelvic physical examination added approximately two minutes to the gynecological examination. The VAMP scores were evaluated in relation with patient age, body weight, and deliveries in both groups, in relation to pelvic diaphragm comorbidities and type of pain felt by the study group.

Statistical analyses were performed using Statistica v 9.1, provided by Statsoft Polska, with population parameters (mean and standard deviation), $\mathrm{x} 2$ test, one-sided t-test, Spearman Correlation and Mann-Whitney $U$ test for critical value $p=0.05$ application.

\section{RESULTS}

Among the 650 women who were included in this study, 449 (69.1\%) women comprised the Vd group and 201 (30.9\%) comprised the control group. According the Vd subtype differentiation [1], half of the Vd cohort suffered from mixed $\mathrm{Vd}$ (50.1\%, 225 women), followed by provoked Vd (26.7\%, 120 women) and spontaneous Vd (23.2\%, 104 women).

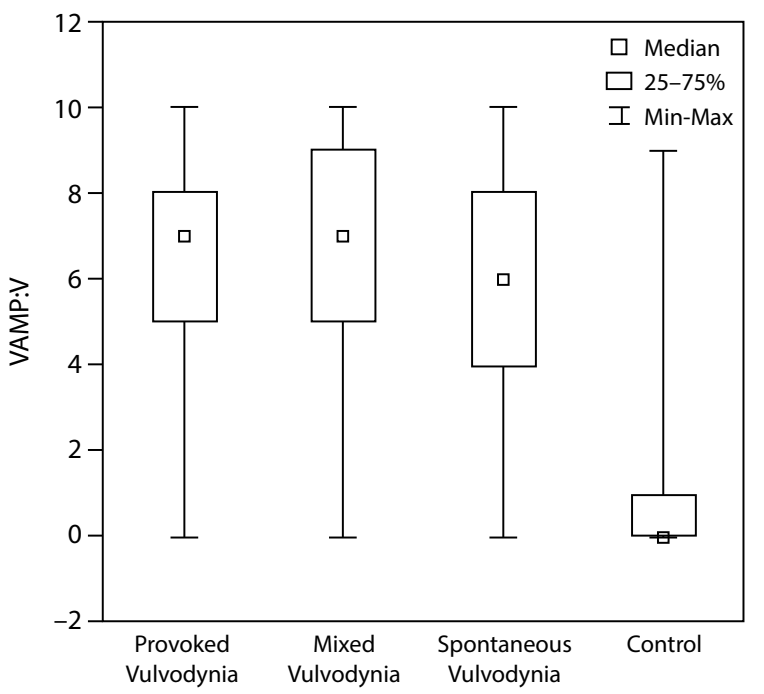

Figure 2. VAMP protocol score: vulvar pain (V) in the three vulvodynia subgroups and the control group

The individuals in the study and the control group differed slightly in age (34.7 years vs 37.6 years old) and body weight $(61.3 \mathrm{~kg}$ vs $66.0 \mathrm{~kg})(\mathrm{Z}=-3.256 ; \mathrm{p}<0.001)$.

Differences in the VAMP protocol scores were identified between the $\mathrm{Vd}$ and the control comparisons in features $\mathrm{V}$ (vulva) $(6.48 \pm 2.64$ vs $0.98 \pm 1.96), \mathrm{M}$ (muscles) $(6.29 \pm 2.55$ vs $1.05 \pm 1.90)$, and $P$ (paraurethral area) $(6.89 \pm 2.37$ vs $1.33 \pm 2.17)$, with $p<0.001$ for each of above values. The $\mathrm{Vd}$ and comparative cohort did not differ in the $A$ (anus) item $(0.03 \pm 0.37$ vs $0.08 \pm 0.57$, respectively, $p=0.11)$. There were also no differences in the VAMP scores between the three vulvodynia subtypes (provoked, mixed, and spontaneous) (Fig. 2-4).

Regression analysis showed no differences in the VAMP ratings in relation to age, body weight, or cesarean delivery. Patients who underwent vaginal deliveries presented with 


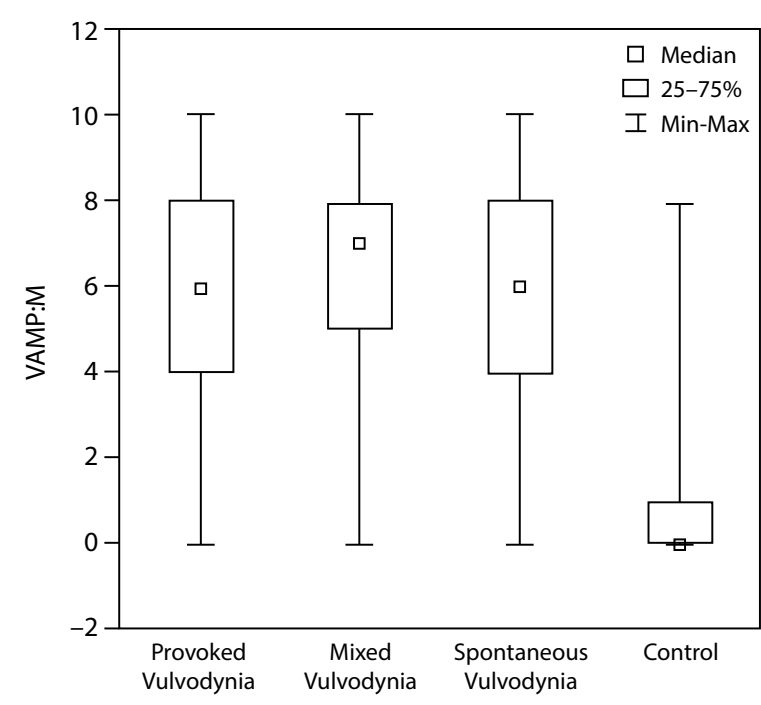

Figure 3. VAMP protocol score: muscle pain (M) in the three vulvodynia subgroups and the control group

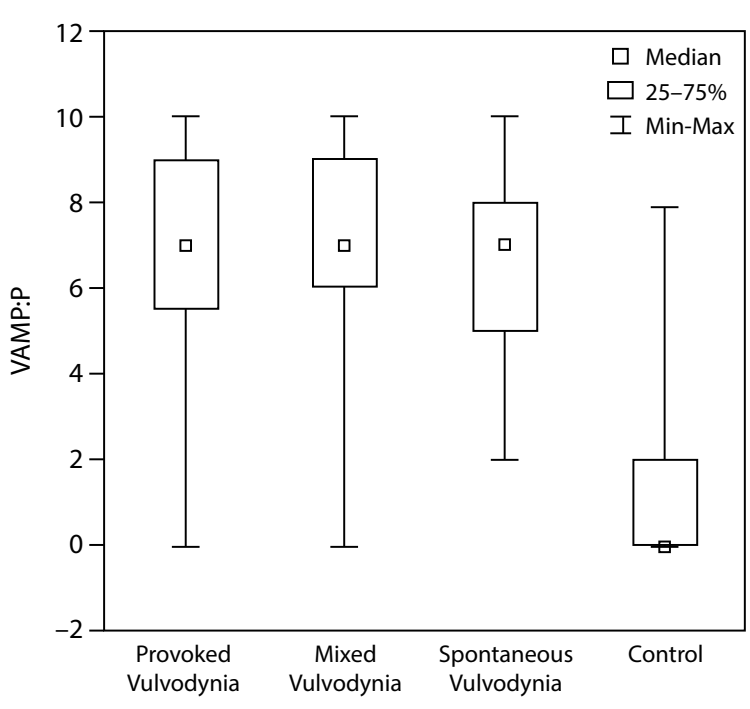

Figure 4. VAMP protocol score: paraurethral area pain $(\mathrm{P})$ in the three vulvodynia subgroups and the control group

Table 1. VAMP protocol score correlation with age, body weight, and vaginal and cesarean delivery in the study (Vd) and control group, based on the regression analysis

\begin{tabular}{|l|l|l|l|}
\hline VAMP Feature & V & M & P \\
\hline Vulvodynia & $p \leq 0.001^{* * *}$ & $p \leq 0.001^{* * *}$ & $p \leq 0.001^{* * *}$ \\
\hline Age & $p=0.481$ (NS) & $p=0.179$ (NS) & $p=0.689$ (NS) \\
\hline Body weigh & $p=0.475$ (NS) & $p=0.493$ (NS) & $p=0.256$ (NS) \\
\hline Vaginal delivery & $p=0.036^{*}$ & $p=0.310$ (NS) & $p=0.256$ (NS) \\
\hline Cesarean delivery & $p=0.737$ (NS) & $p=0.500$ (NS) & $p=0.968$ (NS) \\
\hline
\end{tabular}

$\mathrm{V}$ - vulva; $\mathrm{M}$ - muscles; $\mathrm{P}$ - paraurethral area; NS - not significant

lower V scores (Tab. 1), and this was the only significant correlation, other than Vd diagnosis, in all of the groups.

On the basis of statistical data, VAMP protocol score cut-off (NRS on pressure) was defined according to consecutive features: $V$, score $2 ; A$, not relevant; $M$, score 3 ; and $P$, score 3 , which differed between the study and the healthy control, comparison group. Based on the ROC curve analysis, there were no differences between VAMP features in the study cohort ( $\mathrm{pV}-\mathrm{M}=0.959, \mathrm{pV}-\mathrm{P}=0.647, \mathrm{pM}-\mathrm{P}=0.539$ ).

Women with Vd showed stable $P$ scores ( $R$ Spearman $=0.074 ; p=0.126$ ) that were independent of the duration of pain, whereas $V$ scores ( $R$ Spearman $=0.136$; $p=0.005)$ and $M$ scores (R Spearman $=0.104 ; p=0.032$ ) increased with persistent symptoms, although the correlation was very weak.

Type of pain and discomfort were divided in Vd women: 1 , pain without specific description (raw or vague) in $34.5 \%$ (155 women); 2 , burning sensation in $50.1 \%$ (225 women); 3 , stabbing, pressure, and pulling pain in $10.9 \%$ (49 women); 4, pruritus in 42.1\% (189 women); and 5, dryness, discomfort, and oversensitivity in $23.2 \%$ (104 women), in the study co- hort. When compared with the VAMP features, the highest V scores $(Z=-4.01 ; p<0.001)$, M scores $(Z=-3.51 ; p<0.001)$, and $P$ scores $(Z=-3.66 ; p<0.001)$ were observed in relation to pain (description 1). In the remaining symptoms (descriptions $2,3,4)$ VMP scores were insignificant for $V d$ group (range from $Z=-0.27, p=0.7$ to $Z=0.1, p=0.92$ ). Lower M scores $(Z=2.30 ; p=0.021)$ were associated with dryness, discomfort, and vulvar oversensitivity (description 5).

No differences in VAMP protocol scores were found in relation to pelvic diaphragm symptoms coexisting with $\mathrm{Vd}$ (Tab. 2).

\section{DISCUSSION}

In our study cohort, half of the women with Vd presented a mixed (both spontaneous and provoked symptoms) $\mathrm{Vd}$ subtype, $25 \%$ presented provoked, and $25 \%$ presented spontaneous. This differs from previous reports in the literature, where provoked Vd has been reported as the most prevalent subtype (64.8\%), followed by spontaneous (20.3\%) and mixed $\mathrm{Vd}$ (14.9\%) [2]. The differences may be attributed to the detailed patient history that was taken, as even mild 
Table 2. Mann-Whitney U test comparison of VAMP protocol values with comorbidities in the study (Vd) and control group

\begin{tabular}{|l|l|l|l|l|l|}
\hline Symptoms & \% cohort & Nr of woman & $\mathbf{V}$ & $\mathbf{M}$ & $\mathbf{P}$ \\
\hline Vd and dysuria & 35.2 & 158 & $\mathrm{p}=0.124$ (NS) & $\mathrm{p}=0.660$ (NS) & $\mathrm{p}=0.083$ (NS) \\
\hline $\begin{array}{l}\text { Vd and bowel/anal } \\
\text { symptoms }\end{array}$ & 42.8 & 192 & $\mathrm{p}=0.702$ (NS) & $\mathrm{p}=0.330$ (NS) & $\mathrm{p}=0.218$ (NS) \\
\hline $\begin{array}{l}\text { Dysuria in control } \\
\begin{array}{l}\text { Bowel/anal } \\
\text { symptoms in control }\end{array}\end{array}$ & 13.1 & 24 & $\mathrm{p}=0.217$ (NS) & $\mathrm{p}=0.063$ (NS) & $\mathrm{p}=0.098$ (NS) \\
\hline
\end{tabular}

Vd - vulvodynia; V - vulva; M - muscles; P — paraurethral area; NS — not significant

recurrent vulvar itch, irritation, or discomfort (e.g., the dryness or oversensitivity found in one fourth of women with $\mathrm{Vd}$ ) were considered as spontaneous symptoms in patients with provoked Vd, which satisfied mixed Vd subtype criteria.

Vague or raw pain symptoms were found in one third of the patients with $\mathrm{Vd}$, although different unpleasant vulvar sensations (stabbing, pulling, pressure, and irritation) were equally found in the study group. The most frequent descriptor of vulvar pain was a burning vulvar sensation (half of the women with Vd), followed by vulvar itch (42.1\%). Mistakenly, these items are often not considered as pain by healthcare professionals or the affected patients. Vd diagnostic criteria, including history taking and physical (gynecological and pelvic) examination, have to be introduced for such patients with recurrent vulvar symptoms.

In the process of diagnosing patients with $\mathrm{Vd}$, a few consecutive steps have to be undertaken $[3,6,9,13]$, in agreement with the study protocol. PFM status examinations were used as a diagnostic tool for every patient with $\mathrm{Vd}$ and were proven useful in distinguishing approximately $80-90 \%$ of patients who benefit from pelvic manual and general physiotherapy, indicated for spontaneous and provoked Vd with PFM dysfunction [23-25]. The main feature of overactive PFM is pain upon touching the vulva (referred pain) and deep muscles upon internal (vaginal or rectal) access [15]. Thus far, no physical pelvic examinations for pelvic floor myofascial pain syndrome assessments have been standardized [18]. Women with prior vaginal delivery presented less vestibulum sensitivity on pressure (lower V score in both study and control group). Regarding to vulvar pain, vulvar score $(\mathrm{V})$ and paraurethral pain on pressure $(P)$ score were higher in patients with vague, raw pain than in those with other pain descriptions. This could be because certain types of pain per se might be more troublesome for patients, and ischemic pain is often described as stabbing, burning, heavy, exhausting and results in lower pain thresholds that are consistent with peripheral sensitization [26].

Since the IMAP research and publications in $2015[13,19]$, where three maps were proposed for pelvic examinations in patients with Vd, a short, modified version (the VAMP protocol) has entered clinical practice and research in outpatient clinic in diagnosing chronic vulvar pain.
Pain on pressure in VAMP protocol scores were highly significant in patients with $\mathrm{Vd}$, according three features: $V, M$ and $P$. This reflects the importance of the three particular referred trigger points for PFM overactive dysfunction. The paraurethral area has to be emphasized as a diagnostic anatomical region.

The anal sphincter area did not experience pain on touch, as noticed in previous publications [19], in both groups, and thereby was excluded from further statistical analyses. Even though the anal cotton swab pressure test (A feature in VAMP) was advised by the study authors, to concentrate the physician's attention on that particular area and to identify the presence of serious pain sources such as anal fissure, lichen simplex or hemorrhoids, especially "red flags" such as anal cancer, should not to be overlooked. The second reason why the anal area should be tested is to objective the overestimation of all VAMP protocol scores due to catastrophism or intentional patient's pain simulation, describing as painful area on pressure.

The relevant level of PFM pain on examination (as the $M$ feature in the present study) is consistent with other reports that used a cut-off pain score of 3 , where pain $\leq 3$ was considered not clinically significant and pain scores $>3$ were deemed significant [27]. In another publication, pelvic floor myofascial pain was determined by transvaginal palpation of the bilateral obturator internus and levator ani muscles, and, in NRS, it was categorized as none (0), mild (1-3), moderate (4-6), or severe (7-10) for each site [25]. Scores $\geq 3$ (in NRS on examination) on at least one area ( $V \geq 3, M \geq 3$, or $P \geq 3$ ) confirmed PFM overactive dysfunction in this study. The three VAMP examination areas can give a broader overactive PFM status estimation than the cotton swab test alone, to improve reliability, as internal consistency of $\mathrm{Vd}$ assessment method.

Based on the systematic review of the pelvic physical examination techniques used to assess pelvic floor myofascial pain in women, no specific technique has been standardized yet, although the key points have been emphasized [18]. Our search for professional pelvic floor muscles dysfunction assessments that are commonly used to validate patient-reported pelvic symptoms identified the Pelvic Floor Distress 
Inventory-20, Pelvic Floor Impact Questionnaire, and Pelvic Organ Prolapse Incontinence Sexual Questionnaire are considered as recommended materials $[11,16]$. The PFM status diagnostic methods that are useful for clinical practice are mostly dedicated to underactive PFM dysfunction assessment $[11,16]$. Overactive PFM dysfunction estimation methods based on physical examination require simplification, description for clinical and educational purposes and further validation.

The study limitation concerns the examination according to VAMP protocol were performed unblinded without a calibrated method of applied pressure, which results from the simplification of the diagnosis protocol for clinical practice. Another weakness of presented method is subjective character of results reported by the patients during examination, although perceived pain is always considered as subjective.

The consciousness of women to PFM problems have grown with time, along with the demand for healthcare professionals in the chronic vulvar pain diagnosis and treatment field. The study provides more detailed information to explain vulvodynia management complex issue, method for gathering data on sensitive and neglected woman health problem. The examination according to VAMP protocol data collection is not time consuming, performed as a part of bimanual exam of woman with persistent vulvar pain. According to the literature and authors experience, the confirmation of an overactive PFM status is crucial for further management through which manual therapy and biofeedback can be applied $[10,13,18]$. Our study suggests that VAMP protocol examination can be useful clinical tool for diagnostic and educational purposes. There is a need for perspective, multicenter studies to confirm VAMP protocol usefulness among physicians dealing with Vd patients.

\section{CONCLUSIONS}

Considering the high prevalence of pelvic floor myofascial pain in $\mathrm{Vd}$ patients a routine assessment for pelvic floor dysfunction should be followed for all patients who present for chronic vulvar and pelvic pain [9, 25]. Pelvic examination according to VAPM protocol can be applied in vulvar pain patients for diagnostic purposes. Besides vulvodynia symptoms, no other analyzed variables had influence on scores of VAMP protocol. We found the cut-off score $\geq 3$, even in one of $\mathrm{V}, \mathrm{M}$ or P component of VAMP protocol, can be considered as diagnostic criterium for Vd with PFM overactivity. Component $\mathrm{A}$ (anus area) was not useful for Vd diagnosis, although remains useful for educational purposes. The VAMP schedule is proposed to be a simple exam protocol that consists of a cotton swab test of the vestibulum, anus touch (although the anus does not experience pain upon applying pressure), pelvic floor muscles evaluation by "finger both sides with pressure motions" and finally, the application of pressure to both sides of the periurethral area. The procedure can be performed during a gynecological exam for Vd diagnosis in chronic vulvar pain women.

\section{Acknowledgments}

None

\section{Funding}

There has been no external financial support for this work.

\section{Conflict of interest}

The authors confirm that there are no known conflicts of interest associated with this publication.

\section{Ethics approval}

IRB approval KE-0254/111/2020, Medical University of Lublin, Poland

\section{REFERENCES}

1. Bornstein J, Goldstein AT, Stockdale CK, et al. consensus vulvar pain terminology committee of the International Society for the Study of Vulvovaginal Disease (ISSVD), the International Society for the Study of Women囚s Sexual Health (ISSWSH), and the International Pelvic Pain Society (IPPS). 2015 ISSVD, ISSWSH, and IPPS Consensus Terminology and Classification of Persistent Vulvar Pain and Vulvodynia. J Low Genit Tract Dis. 2016; 20(2): 126-130, doi: 10.1097/LGT.0000000000000190, indexed in Pubmed: 27002677.

2. Reed B, Harlow S, Sen A, et al. Prevalence and demographic characteristics of vulvodynia in a population-based sample. Am J Obstet Gynecol. 2012; 206(2): 170.e1-170.e9, doi: 10.1016/j.ajog.2011.08.012.

3. Jantos MA. Myofascial Perspective on Chronic Urogenital Pain in Women. In: Santoro GA, Wieczorek AP, Bartram C. ed. Pelvic Floor Disorders 2nd ed. Springer : in press.

4. Butrick CW. Pelvic floor hypertonic disorders: identification and management. Obstet Gynecol Clin North Am. 2009; 36(3): 707-722, doi: 10.1016/j.ogc.2009.08.011, indexed in Pubmed: 19932423.

5. Pukall CF, Binik YM. Vulvodynia. In: Mayer EA, Bushnell MC. ed. Functional Pain Syndromes: presentation and pathophysiology. IASP Press, Seattle 2009: 71-81.

6. Harlow BL, Stewart EG. A population-based assessment of chronic unexplained vulvar pain: have we underestimated the prevalence of vulvodynia? J Am MedWomens Assoc (1972). 2003; 58(2): 82-88, indexed in Pubmed: 12744420.

7. Messelink B, Benson T, Berghmans B, et al. Standardization of terminology of pelvic floor muscle function and dysfunction: report from the pelvic floor clinical assessment group of the International Continence Society. Neurourol Urodyn. 2005; 24(4): 374-380, doi: 10.1002/nau.20144, indexed in Pubmed: 15977259.

8. Nunns D, Mandal D, Byrne M, et al. British Society for the Study of Vulval Disease (BSSVD) Guideline Group. Guidelines for the management of vulvodynia. Br J Dermatol. 2010; 162(6): 1180-1185, doi: 10.1111/j.136 5-2133.2010.09684.x, indexed in Pubmed: 20331460.

9. Goldstein AT, Pukall CF, Brown C, et al.Vulvodynia: Assessment and Treatment. J Sex Med. 2016; 13(4): 572-590, doi: 10.1016/j.jsxm.2016.01.020, indexed in Pubmed: 27045258.

10. Gentilcore-Saulnier E, McLean L, Goldfinger C, et al. Pelvic floor muscle assessment outcomes in women with and without provoked vestibulodynia and the impact of a physical therapy program. J Sex Med. 2010; 7(2 Pt 2): 1003-1022, doi: 10.1111/j.1743-6109.2009.01642.x, indexed in Pubmed: 20059663.

11. Haylen BT, de Ridder D, Freeman RM, et al. An International Urogynecological Association (IUGA)/International Continence Society (ICS) joint report on the terminology for female pelvic floor dysfunction. Int Urogynecol J. 2010; 21(1): 5-26, doi: 10.1007/s00192-009-0976-9, indexed in Pubmed: 19937315. 
12. Reissing ED, Brown C, Lord MJ, et al. Pelvic floor muscle functioning in women with vulvar vestibulitis syndrome. J Psychosom Obstet Gynaecol. 2005; 26(2): 107-113, doi: 10.1080/01443610400023106, indexed in Pubmed: 16050536.

13. Jantos $M$, Johns $S$, Torres $A$, et al. Mapping chronic urogenital pain in women: rationale for a muscle assessment protocol - the IMAP, Part 1. Pelviperineol. 2015; 34: 21-27.

14. Jantos M, Burns NR. Vulvodynia. Development of a psychosexual profile. J Reprod Med. 2007; 52(1): 63-71, indexed in Pubmed: 17286072

15. Sarton J. Assessment of the pelvic floor muscles in women with sexual pain. J Sex Med. 2010; 7(11): 3526-3529, doi: 10.1111/j.1743-6109.201 0.02059.x, indexed in Pubmed: 21064249.

16. AguilarVC, White AB, Rogers RG. Updates on the diagnostic tools for evaluation of pelvic floor disorders. Curr Opin Obstet Gynecol. 2017;29(6):458-464, doi: 10.1097/GCO.000000000000415, indexed in Pubmed: 28938376.

17. Zolnoun D, Bair E, Essick G, et al. Reliability and reproducibility of novel methodology for assessment of pressure pain sensitivity in pelvis. J Pain. 2012; 13(9): 910-920, doi: 10.1016/j.jpain.2012.06.006, indexed in Pubmed: 22958875.

18. Meister MR, Shivakumar N, Sutcliffe S, et al. Physical examination techniques for the assessment of pelvic floor myofascial pain: a systematic review. Am J Obstet Gynecol. 2018; 219(5): 497.e1-497.e13, doi: 10.1016/j. ajog.2018.06.014, indexed in Pubmed: 29959930.

19. Jantos M, Johns S, Torres A, et al. Mapping chronic urogenital pain in women: insights into mechanisms and management of pain based on the IMAP, Part 2. Pelviperineol. 2015; 34: 28-36.

20. Davenport RB, Voutier CR, Veysey EC. Outcome Measurement Instruments for Provoked Vulvodynia: A Systematic Review. J Low Genit Tract
Dis. 2018; 22(4): 396-404, doi: 10.1097/LGT.0000000000000418, indexed in Pubmed: 30059352.

21. dos Santos Calderon P, Peixoto RF, Gomes VM, et al. Concordance among different pain scales in patients with dental pain. J Orofac Pain. 2012; 26(2): 126-131, indexed in Pubmed: 22558612.

22. Friedrich EG. Jr. Vulvar vestibulitis syndrome. J Reprod Med. 1987; 32: 110-114.

23. Bo K, Frawley HC, Haylen BT, et al. An International Urogynecological Association (IUGA)/International Continence Society (ICS) joint report on the terminology for the conservative and nonpharmacological management of female pelvic floor dysfunction. Neurourol Urodyn. 2017; 36(2): 221-244, doi: 10.1002/nau.23107, indexed in Pubmed: 27918122

24. Wallace SL, Miller LD, Mishra K. Pelvic floor physical therapy in the treatment of pelvic floor dysfunction in women. Curr Opin Obstet Gynecol. 2019; 31(6): 485-493, doi: 10.1097/GCO.0000000000000584, indexed in Pubmed: 31609735.

25. Meister MR, Sutcliffe S, Badu A, et al. Pelvic floor myofascial pain severity and pelvic floor disorder symptom bother: is there a correlation? Am J Obstet Gynecol. 2019; 221(3): 235.e1-235.e15, doi: 10.1016/j. ajog.2019.07.020, indexed in Pubmed: 31319079.

26. Mense S, Simons DG, Russell IJ. Muscle pain: understanding its nature, diagnosis, and treatment. Lippincott Williams \& Wilkins : 16-18.

27. Kavvadias T, Pelikan S, Roth $\mathrm{P}$, et al. Pelvic floor muscle tenderness in asymptomatic, nulliparous women: topographical distribution and reliability of a visual analogue scale. Int Urogynecol J. 2013; 24(2): 281-286, doi: 10.1007/s00192-012-1837-5, indexed in Pubmed: 22707005 . 